\section{Spinal Cryptococcoma Mimicking Spinal Cord Tumor Complicated by Cryptococcal Meningitis in an Immunocompetent Patient}

\section{Shanthi Viswanathan*, Carol Lai, Suraya Yusoff and Norzaini Rose}

Department of Neurology, Hospital Kuala Lumpur, Jalan Pahang, Kuala Lumpur, 50560, Malaysia

*Corresponding author: Shanthi Viswanathan, Department of Neurology, Hospital Kuala Lumpur, Jalan Pahang, Kuala Lumpur, 50560, Malaysia, Tel: 03-26155400; Fax: 03-26911186; E-mail: shivenda70@yahoo.com

Received date: May 20, 2017; Accepted date: June 26, 2017; Published date: June 30, 2017

Copyright: (c) 2017 Viswanathan S, et al. This is an open-access article distributed under the terms of the Creative Commons Attribution License, which permits unrestricted use, distribution, and reproduction in any medium, provided the original author and source are credited.

\begin{abstract}
There are very few cases in the literature on spinal cryptococcomas occurring alone without cerebral involvement at onset. Although cases of cerebral cryptoccomas followed by arachnoiditis have been reported, cases of spinal cryptococcoma followed by cryptococcal meningitis are exceedingly rare. We present a patient with spinal cord cryptococcoma who subsequently developed cryptococcal meningitis in a young immunocompetent patient. Early recognition and aggressive anti-fungal treatment is important.
\end{abstract}

Keywords: Spinal cryptococcoma; Cryptococcal meningitis

\section{Introduction}

Cryptococcus is an encapsulated yeast, which allows them to have antiphagocytic and immunosuppresive abilities. There are over 50 species, but only $C$. neoformans var. neoformans ( $C$. neoformans) and $C$. neoformans var. gattii ( $C$. gatti) are considered principal pathogens in humans [1] and one of the most common fungal infections in the central nervous system [1]. C. neoformans (serotypes A, D and AD) and $C$. gattii (serotypes $\mathrm{B}$ and $\mathrm{C}$ ) are the commonest subtypes.

C. neoformans is the most common species in the United States and other temperate climates throughout the world and is found in aged pigeon droppings [1,2]. C. gattii is mainly found in the tropics but is not associated with birds; it grows in certain species of eucalyptus trees (i.e., Eucalyptus camaldulensis, Eucalyptus tereticornis). $C$. neoformans causes cryptococcal infections in immunocompromised patients, whereas $C$. gatti usually infects immunocompetent patients [1-5].

The cryptococcal spores are inhaled by humans and get lodged in the lungs. During periods of impaired immunity, the spores disseminate to other organs especially the central nervous system (CNS). Meningitis is the commonest manifestation of cryptococcosis of the CNS [1-7]. There are very few cases in the literature on spinal cryptococcomas occurring alone without cerebral involvement at onset. Although cases of cerebral cryptoccomas followed by arachnoiditis have been reported, cases of spinal cryptococcoma followed by cryptococcal meningitis are exceedingly rare [3-6]. We present a young immunocompetent male patient with spinal cord cryptococcoma who subsequently developed cryptococcal meningitis.

\section{Case Report}

A 19 year old adolescent presented with acute onset of weakness of the lower limbs following a fall at home. He was admitted to a neighbouring state hospital and referred to our centre for further management after three weeks of symptoms. There was no history of prior weakness of the lower limbs or sphincter involvement and patient was in good health. General physical examination revealed an alert and oriented young man.

Neurological examination of the upper limbs was normal. Lower limb examination revealed increased tone and clonus bilaterally. Power over the lower limbs were Medical research council scale (MRC) 3/5 on the left and MRC 1/5 on the right with extensor plantar responses bilaterally. Pin-prick sensation, proprioception and vibration were reduced till the level of L1 bilaterally. There were no signs of meningeal irritation.

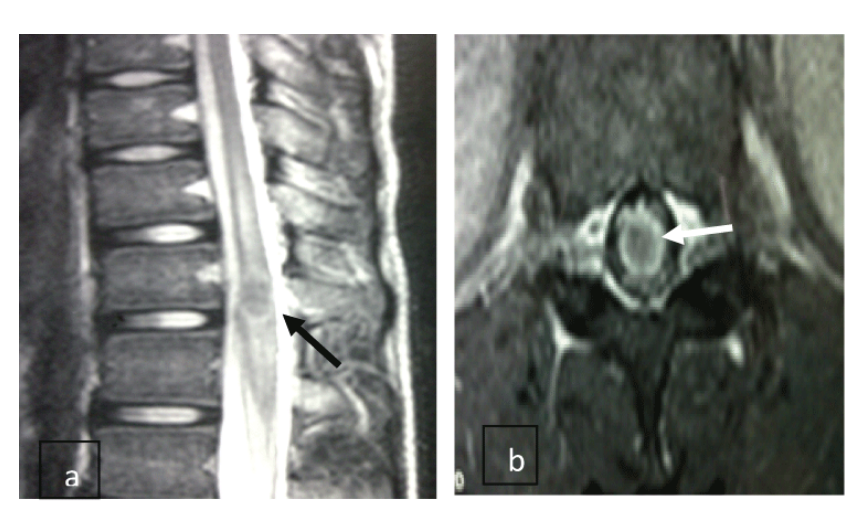

Figure 1: (a) Sagittal T2WI MRI of the spinal cord showing a ring like intramedullary lesion within the thoracic spinal cord. The lesion had central hypointensity with surrounding cord oedema (arrow). (b) Axial T1 MRI with contrast of spinal cord showing a ring enhancing lesion within the thoracic cord.

Blood tests including full blood count, erythrocyte sedimentation rate, tumour markers, HIV and viral hepatitis screens were normal. Chest X-ray and X-ray of the thoraco-lumbosacral spine were unremarkable. Urgent MRI of the whole spine \{Sagittal T2 weighted imaging (WI)\} showed a ring-like intramedullary lesion within the thoracic cord asymmetrically occupying the entire central, lateral and posterior portions of the cord. The lesion had a central hypointensity with surrounding cord oedema and ring enhancement post contrast. 
The lesion was initially suspected to be a spinal cord tumour, possibly an ependymoma (Figures 1a and $1 \mathrm{~b}$ ).

The patient was operated within $48 \mathrm{~h}$ of hospitalisation to our tertiary hospital. T11 laminoplasty and excision of the tumour was performed. Post operatively, the patient's general consciousness remained the same. However, the power of the lower limbs deteriorated to MRC $2 / 5$ on the left and MRC $0 / 5$ on the right side. He was diagnosed with post-operative oedema, and was given dexamethasone $4 \mathrm{mg}$ eight hourly. One week later, there was improvement of the lower limb power to $3 / 5$ bilaterally.

Biopsy of the cord lesion surprisingly showed granulomas, multinucleated giant cells, lymphocytes with central necrosis and encapsulated organisms positive for periodic acid Schiff (which stains for the cell wall and body of fungal elements) and Gomorri methenamine silver (GMS) stain (which stains exclusively for fungal organisms causing cell walls to stain black or brown). These histopathological changes,combined with the findings on histochemical stains and subsequent cultures were consistent with chronic granulomatous inflammation secondary to $C$. neoformans infection though the patient did not have any signs and symptoms of systemic fungal infection. (Figures 2a-2c) Antifungal treatment with intravenous amphotericin and fluconazole was started rather than Flucytosine which has been reported to result in quicker clearance of viable yeasts from the central nervous system as the latter drug was not readily available at our center that time.

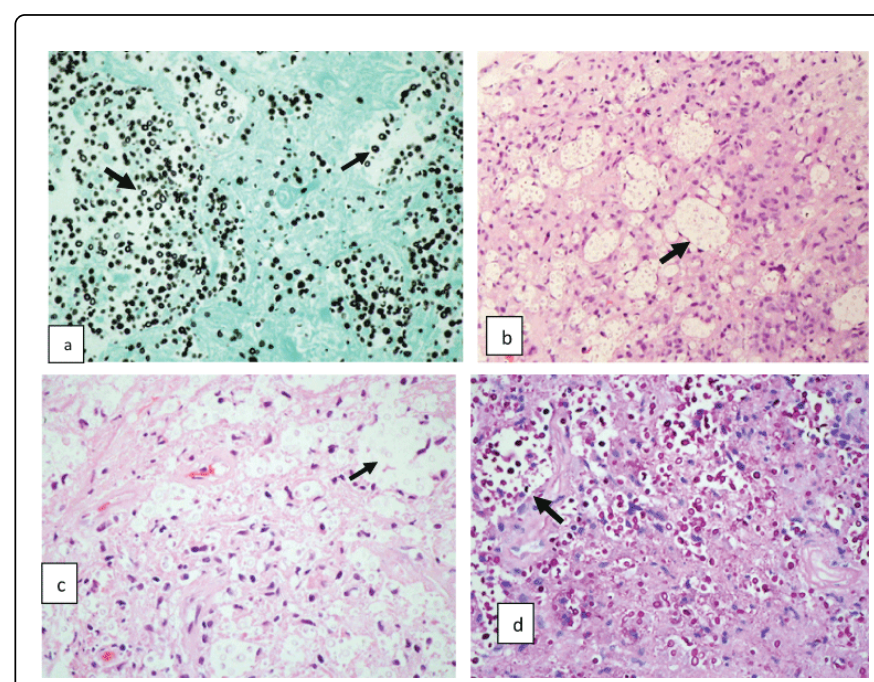

Figure 2: Histopathology of spinal cord lesion, (a-d) showing Gomori methenamine silver (GMS) and Periodic acid schiff stains (PAS) (a,d) and Hemotoxylin and eosin stain at 400x \& 600x power $(\mathrm{c}, \mathrm{d})$ of the spinal cord lesion showing (arrows) granulomas, multinucleated giant cells and encapsulated organisms (thickened membranes) positive for PAS and GMS stains.

On the $13^{\text {th }}$ day postoperatively, the patient complained of headache and vomiting followed by two episodes of generalized tonic-clonic seizures. Glasgow coma scale (GCS) dropped to 8/15 (Eye 3, Motor 5, Verbal 2). Examination revealed bilateral papilloedema and bilateral sixth nerve palsies. The power was MRC $3 / 5$ at both upper limbs and $1 / 5$ over both lower limbs with generalised hypertonia, hyper reflexia and up going plantar responses bilaterally.
Urgent CT brain (plain) showed early non-obstructive hydrocephalus (Figure 3). Lumbar puncture done revealed raised cerebrospinal fluid (CSF) opening pressure of $>30 \mathrm{~cm} \mathrm{H}_{2} 0$ with high CSF protein $(1.0 \mathrm{~g} / \mathrm{dL})$ and low CSF sugars $(0.6 \mathrm{mg} / \mathrm{dL})$. CSF Indian Ink for Cryptococcus was positive for encapsulated yeasts and CSF cryptococcal antigen was positive. CSF cryptococcal culture grew $C$. neoformans. The patient was treated aggressively with intravenous mannitol, lumbar punctures, neurosurgical intervention, intravenous amphotericin B and fluconazole. However, eventually, the patient developed nosocomial sepsis with prolonged hospitalization leading to a fatal outcome after one month despite aggressive antifungal treatment. Early recognition of spinal cord cryptococcomas with development of cryptococcal meningitis as a delayed complication is important.

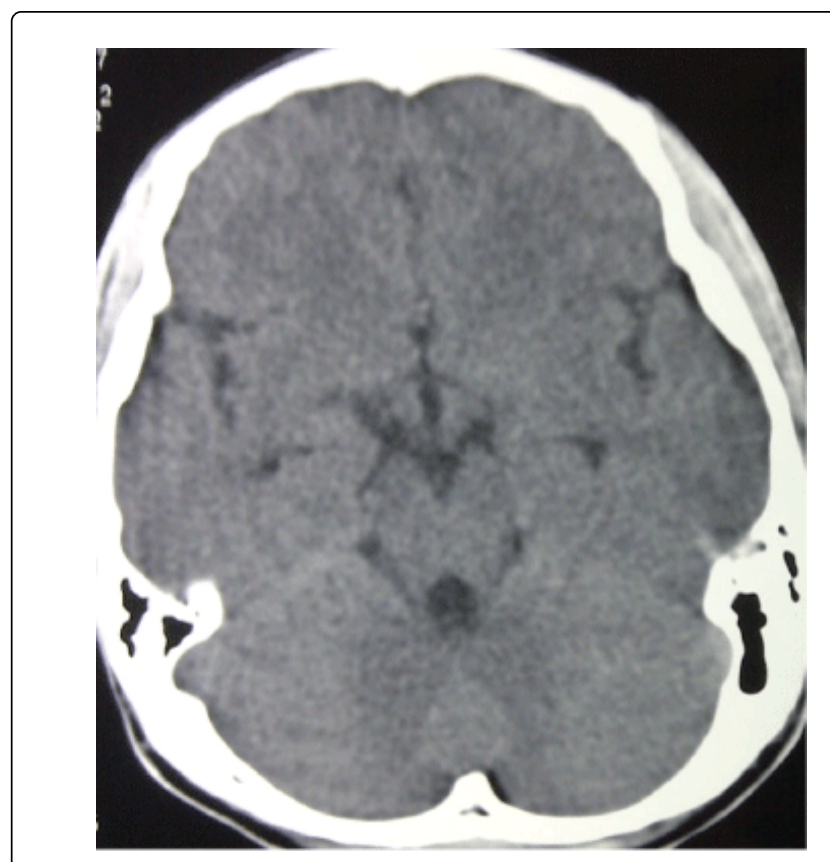

Figure 3: Axial CT brain done post laminectomy showing mild communicating hydrocephalus with temporal horns of the lateral ventricle being seen.

\section{Discussion}

Acute onset presentation of intramedullary spinal cord cryptococcoma is a rare initial presentation of cryptococcal infection. We are describing an unusual case of a patient with a solitary spinal cord cryptococcoma followed by cryptococcal meningitis in a young immunocompetent patient occurring in the absence of cerebral cryptococcomas. Prior to this, he did not have fever, systemic complaints, headaches, vomiting and seizures to suggest central nervous system infection or raised intracranial pressure at the onset. This immunocompetent patient presented acutely with sudden onset of lower limb weakness as a result of the spinal cryptococcoma. Though his eventual outcome was poor, this case highlights important learning points to consider as the initial lesion mimicked a cord tumor in the absence of systemic complaints and the presence of disseminated disease as a complication of the initial pathology. 
Infection with $C$. gattii usually affects immunocompetent patients. It presents with a granulomatous inflammatory response, mainly in tropical and subtropical regions resulting in chronic disease as compared to $C$. neoformans. Subjects infected with $C$. gattii are at risk of intracerebral mass lesions (e.g. cryptococcomas) as well as meningitis, encephalomyelitis and optic nerve atrophy. In comparison, C. neoformans is present worldwide. It has a tendency to cause infection in predominantly immunodeficient patients (90\%) though not exclusively limited to these types of patients, leading to acute meningitis or meningoencphalitis with a higher mortality. Patients with $C$. gatti are characterized by less mortality as an outcome but with neurological sequelae, the need for surgery and prolonged therapy [1-5]. In our case, $C$. neoformans infection of the spinal cord developed in an otherwise immunocompetent young man which again is against what is commonly reported in the literature. This underscores certain differences in presentation locally in Malaysia. The subject progressed to develop cryptococcal meningitis despite antifungal therapy and surgical intervention possibly due to dissemination of disease.

The intramedullary lesions of spinal cryptococcosis are described as cryptococcomas [1-8]. Intramedullary spinal cryptococcomas are less common compared to intradural and other types of extradural lesions [8]. In the literature there have been a few reports of spinal intramedullary cryptococcomas. In these rare reports, the majority presented with paraparesis and pyramidal signs in the lower limbs with a sensory level in the thoracolumbar region [6-8]. Shen et al. reported a similar rare case of a conus medullaris cryptococcoma in an immunocompetent host in the absence of meningoencephalitis with symptoms mimicking an intramedullary spinal cord tumor. Their patient had a good outcome with surgical removal in the absence of dissemination of the lesion followed by post-operative anti-fungal treatment [6]. As in our case, the lesion was initially thought to be a tumor. Majority developed the lesions in the thoracic or upper lumbar regions and were diagnosed after a biopsy [6-8]. The cryptococcomas on MRI spine appeared as hyperintense lesions with oedema and ring enhancement, similar to our patient. The survival rate for these patients if limited to the spine was high [6-10]. The incidence of spinal cryptococcomas with cerebral meningitis is not well documented. In one of the earliest reports by Grosse et al., a young female patient developed lumbar myelitis due to an intramedullary lumbar cryptoccoma and cerebral cryptococcomas necessitating combined therapy with antifungals, steroids (for the myelitis) and decompressive laminectomy which produced overall good outcome after 2 years of antimycotic treatment [3]. Aside from this early study, Stein et al. [12] reported four patients with mycotic spinal arachnoiditis and meningitis due to cryptococcal infection. Three of these patients developed acute fungal meningitis postoperatively and two out of the three died, similar to our case. None of them had cryptococcomas of the spine. Therefore mortality is higher when cerebral involvement is present [6-12]. In our patient the overall outcome despite correct diagnosis, dissemination of the disease and institution of aggressive antimycotic treatment was complicated by nosocomial septicaemia. In order to improve therapeutic outcomes, there have been a number of reports suggesting the possible benefits of intravenous amphotericin in combination with intrathecal amphotericin B. In a trial conducted by Yuchong et al., all 14 patients survived with no evidence of relapse during 1 year follow-up with intrathecal injection of amphotericin B or with low-dose therapy [13]. Therefore, this technique should be considered as it appears to be effective and safe in carefully selected patients though it was not tried in our patient.
Steroids were used early in this case in the presence of mass effect to help reduce the swelling caused by the tumor residing at the spinal region which was causing significant neurological deficits. There is a lack of strong evidence showing the risks or benefits of steroid use in severe CNS cryptococcal disease. However, there are multiple case reports including the case described by Grosse $\mathrm{P}$ et al mentioned earlier in this article [3-11]. As in the latter case, therapeutic success and good neurologic outcomes were achieved with the use of adjunctive steroids in non-HIV infected individuals with severe CNS cryptococcal infection especially when complicated by cryptococcomas in the cerebral cortex and spine to reduce the inflammatory response [6-14]. Nonetheless, more randomised control trials are needed to elucidate the cautious benefits versus the risks.

Early shunting by aggressive neurosurgical intervention has also been advocated by consensus guidelines even when the ventricles are not that large in order to improve outcomes and prevent the discomfort experienced by patients due to repeated lumbar punctures. In fact a number of studies have shown the benefits in terms of morbidity and mortality outcomes. The placement of a ventriculoperitoneal shunt, even in cases where the neuroimaging studies do not reveal presence of a hydrocephalus and in those with persistent CSF cryptococcal infection can address the problem of raised intracranial pressures [14]. Furthermore, depending on the response to medical treatment, decompressive laminectomy with local expansion of the dura also can be considered to prevent irreversible spinal cord damage as was done in this case.

In summary, we are presenting an interesting and rare case of an immunocompetent patient with spinal intramedullary cryptococcoma followed by acute cryptococcal meningitis postoperatively. Although rare, spinal cryptoccomas should be thought of in patients from endemic regions and when the MR imaging shows hyperintense lesions with ring enhancement involving thoracic and upper lumbar region [9]. This diagnosis needs to be entertained in young patients, as this disease is potentially treatable.

\section{Informed Consent}

Informed consent was taken for the purpose of publication of this case report, images and biopsy results.

\section{Acknowledgement}

The publication of this article is part of the approval obtained from the institutional review board for collection of data from the demyelinating database MREC 10503 for collection of data on myelitis, optic neuritis, multiple sclerosis, neuromyelitis optica spectrum disorders and publication of case reports.

\section{Conflicts of Interest}

The authors have no conflicts of interest.

\section{Authors Contributions}

Study concept and design: Dr. Shanthi Viswanathan and Dr. MeiLing Sharon TAI. Acquisition, analysis and interpretation of data by Dr. Shanthi Viswanathan, Dr. Sharon Tai, Dr. Norzaini Rose and Dr. Suryati Mohd Yusoff. Drafting of paper by Dr. Shanthi Viswanathan and Dr. Mei-Ling Sharon TAI. Revision of paper for important intellectual content by Dr. Shanthi Viswanathan, Dr Mei-Ling Sharon 
Citation: Viswanathan S, Lai C, Yusoff S, Rose N (2017) Spinal Cryptococcoma Mimicking Spinal Cord Tumor Complicated by Cryptococcal Meningitis in an Immunocompetent Patient. J Neurol Neurophysiol 8: 432. doi:10.4172/2155-9562.1000432

Page 4 of 4

Tai, Dr. Suryati Mohd Yusoff. Study supervision: Dr. Shanthi Viswanathan.

\section{References}

1. King JW (2016) Cryptococcosis treatment and management: Medscape.

2. Casadevall A, Steenbergen JN, Nosanchuk JD (2003) 'Ready-made' virulence and 'dual use' virulence factors in pathogenic environmental fungi-the Cryptococcus neoformans paradigm. Curr Opin Microbiol 6: 332-337.

3. Speed B, Dunt D (1995) Clinical and host differences between infections with two varieties of Cryptococcus neoformans. Clin Infect Dis 21: 28-34.

4. Grosse P, Tintelnobt K, Söllnerc O, Schmitza B (2001) Encephalomyelitis due to Cryptococcus neoformans var. gattii presenting as spinal tumour: Case report and review of the literature. J Neurol Neurosurg Psychiatry 70: 113-116.

5. Ramamurthi B, Anguli VC (1954) Intramedullary cryptococcic granuloma of spinal cord. J Neurosurg 11: 622-624.

6. Shen CC, Cheng WY, Yang MY (2006) Isolated intramedullary cryptococcal granuloma of the conus medullaris: Case report and review of the literature. Scand J Infect Dis 38: 562-565.

7. Lai PH, Wang JS, Chen WL, Pan HB, Yang CF (2001) Intramedullary spinal cryptococcoma: A case report. J Formos Med Assoc 100: 776-778.
8. Deus-Silva L, Costa AE, Bevilacqua JM, Assis DB, Ferraz CA (2004) Meningoradiculitis due to Cryptococcus neoformans in an immunocompetent patient. Arq Neuropsiquiatr 62: 147-149.

9. Su MC, Ho WL, Chen JH (1994) Intramedullary cryptococcal granuloma of spinal cord: a case report. Zhonghua Yi Xue Za Zhi (Taipei) 53: 58-61.

10. Inada T, Imamura H, Kawamoto M, Sekiya H, Imai Y, et al. (2014) Cryptococcus neoformans var. gattii meningoencephalitis with cryptococcoma in an immunocompetent patient successfully treated by surgical resection. No Shinkei Geka 42: 123-127.

11. Gültaşli NZ, Ercan K, Orhun S, Albayrak S (2007) MRI findings of intramedullary spinal cryptococcoma. Diagn Interv Radiol 13: 64-67.

12. Stein CS, Corrado ML, Friedlander BS, Farmer P (1982) Chronic mycotic meningitis with spinal involvement (arachnoiditis): A report of five cases. Ann Neurol 11: 519-524.

13. Yuchong C, Jianghan C, Hai W, Julin G (2011) Lumbar puncture drainage with intrathecal injection of amphotericin B for control of cryptococcal meningitis. Mycoses 54: e248-251.

14. Corti M, Priarone M, Negroni R, Gilardi L, Castrelo J, et al. (2014) Ventriculoperitoneal shunts for treating increased intracranial pressure in cryptococcal meningitis with or without ventriculomegaly. Rev Soc Bras Med Trop 47: 524-527. 